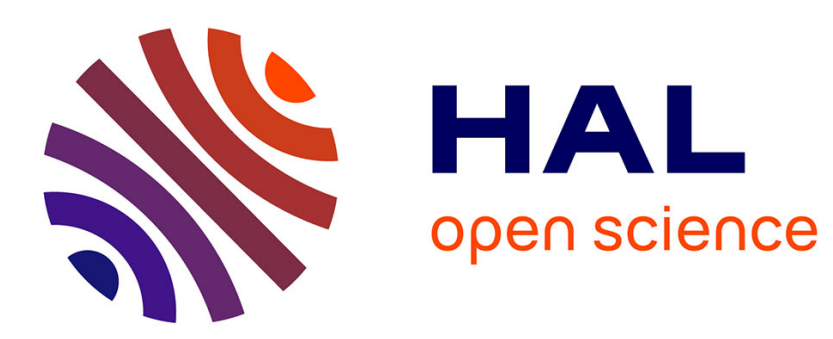

\title{
Workflow Model for Collaborative 3D Interaction - Application to co-located manipulation
}

Christophe Domingues, Samir Otmane, Frédéric Davesne, Malik Mallem

\section{To cite this version:}

Christophe Domingues, Samir Otmane, Frédéric Davesne, Malik Mallem. Workflow Model for Collaborative 3D Interaction - Application to co-located manipulation. International Conference on Computer Graphics Theory and Application (GRAPP 2012), Feb 2012, Rome, Italy. pp.407-412. hal-00652877

\section{HAL Id: hal-00652877 https://hal.science/hal-00652877}

Submitted on 16 Dec 2011

HAL is a multi-disciplinary open access archive for the deposit and dissemination of scientific research documents, whether they are published or not. The documents may come from teaching and research institutions in France or abroad, or from public or private research centers.
L'archive ouverte pluridisciplinaire HAL, est destinée au dépôt et à la diffusion de documents scientifiques de niveau recherche, publiés ou non, émanant des établissements d'enseignement et de recherche français ou étrangers, des laboratoires publics ou privés. 


\title{
WORKFLOW MODEL FOR COLLABORATIVE 3D INTERACTION Application to co-located manipulation
}

\author{
Christophe Domingues ${ }^{1}$, Samir Otmane ${ }^{1}$, Frédéric Davesne ${ }^{1}$ and Malik Mallem ${ }^{1}$ \\ ${ }^{1}$ Univeristy of Evry, France - IBISC EA 4526 \\ \{firstname.lastname\}@ibisc.univ-evry.fr
}

Keywords: Collaboration, 3D Interaction, Multiuser, Virtual Reality, Collaborative Virtual Environments, Workflow

\begin{abstract}
Collaborative Virtual Environments (CVEs) are complex environments where multiple users share the same objects to act together. The complexity of these environments is related to the interaction of the user group with shared items. In a shared world, several constraints appear, including those related to coordination and communication of users and user interaction management in virtual world towards objects and other users. This paper presents a useful workflow-based approach to manage 3D interactions and enhance presence in CVEs. This approach combines astutely different concepts from two research domains: CSCW and VR.
\end{abstract}

\section{INTRODUCTION}

The existing single or multiuser 3D interactions in Virtual Reality (VR) are currently far from providing suitable solutions. Indeed, the 3D interaction suffers from a lack of models and formalisms to manage and control the actions and intentions of users in the virtual environment. The interaction of multiple users with the virtual environment is limited and many researches are in progress. The main objective of the research in the field of multiuser 3D interaction is to instruct users to evolve in CVEs, and to interact together efficiently and easily with virtual entities. Currently, there are two types of synchronous multiuser 3D interaction techniques. A first category separates the degrees of freedom (DoF) for the object to manipulate. In this case, users operate single user techniques by acting on the degrees of freedom which are assigned to them. For the second category, a function will determine the final movement from the position and orientation of the user object in the CVE. This involves using a new technique in multiuser situations. However, all of these techniques do not take into account the constraints of coordination and communication between users and focus exclusively on manipulation tasks with two users.

However, the design of a CVE requires considering multiuser interactions from Computer Supported Collaborative Work (CSCW) point of view, as well as the management of group interactions. Another aspect to consider is the heterogeneity of human machine interface used by participants as well as the disparity between users for providing new models and systems supporting efficient collaborative work and enhancing presence in CVEs. First of all, we present background of recent contributions concerning collaborative 3D interaction techniques and fundamental concepts relevant to the management of group interactions in CVE. Next we quickly present our workflow-based approach. After that, we present the use of the approach for a cooperative manipulation of a virtual object.

\section{RELATED WORK}

\subsection{D Interaction}

In the literature there are two approaches to describe a collaborative 3D interaction [Pinho et al., 2008]. The first allows a simultaneous action on an object by separating data (e.g. degrees of freedom) as rotations and translations to assign them to different users. In this approach, users can act together on the same object but when one user performs translations the other user does only rotations. This approach is used by [Noma et al., 1997]. They interact with a shared object via 
haptic arms. Users are represented by simple virtual hands. The final movement of the object is the result of balance of forces applied by users. Also, we can cite the work of [Duval et al., 2006]. They presented a technique designed to keep the history of interaction and allow the correct representation of simultaneous interactions. More recently, the "Three virtual hands" technique by [Aguerreche et al., 2009] which determines the motion of an object from three points associated to three users' virtual hand. In this technique only translations of virtual hands are taken into account. More recently, [Duval et al., 2009] proposed an asymmetric" $2 \mathrm{~d}$ pointer $/ 3 \mathrm{~d}$ rays" technique for 3D interaction within CVE.

\subsection{Interaction Management}

The best-known work performed for the management of interactions in the CVEs includes the spatial model of interactions proposed by [Greenhalgh, 1999]. This model uses the properties of space as a basis to negotiate interactions and communications between communicating objects. A metric space is defined and used to measure the positions and directions of different objects. From the position and orientation settings, objects have the ability to modify their interaction and communication. Objects interact with each other via a combination of media transmission such as audio, text or visual data through specific interfaces. Recently, a model proposed by [Otmane et al., 2007] is fully dedicated to collaborative $3 \mathrm{D}$ interaction. This model gives to users an ability to have knowledge about the system state and on the other hand provides information needed by the system to assist users to interact together.

\section{WORKFLOW APPROACH}

The main idea of our approach is to disrupt the perception/cognition/action loop by incorporating the concept of workflow. The workflow (WF) manages all the tasks to be performed and all actors involved in the collaboration process. Therefore it can be used on one hand for the coordination of 3D interaction tasks (navigation, selection and manipulation) and on the other hand for the communication of users in the CVE. This functional framework allows users to have knowledge about the system state and other users' activities (who interact or communicate). On the other hand, it must provide information to assist users to interact (easy selection, intend detection) and communicate with others and more generally to work together. The proposed WF consists of two components: a shared and a motor component. The "shared" represents the shared data space that symbolizes the behaviour of users and sources in the CVE. It can be considered as a collective memory in the CVE. This memory will give information about actions of the users. The "motor" represents the set of functions that deal with data processing from the shared space and provides tools to assist the users during the collaboration.

\subsection{Shared component}

The shared component consists of two state matrices representing respectively the state of all users and all sources in the CVE. These two matrices define the overall state of the system and are used to characterize the CVE at any time. These state matrices are constructed from information of users and sources. A source is an object that generates sensory information (virtual object and data media) that can be perceived by the users. Mathematically, we defined a CVE by the triplet, users, sources and workflow. We defined a user as the couple Avatar and State Vector. A user state vector is represented by several elements and it is calculated in real-time:

- User's position and orientation;

- User's force;

- User's avatar speed and virtual weight.

Similarly, the source is defined by the tuple Virtual Object and State Vector. The source state vector is represented by several elements and it is calculated in real-time:

- Source position and orientation;

- Source speed;

- Source virtual weight

Consequently, we can determinate the WF matrices: Users and sources matrix as illustrated on the following equations:

$$
\begin{aligned}
M E_{U} & =\left[\begin{array}{llll}
P_{u_{0}} & \ldots & P_{u_{i}} & P_{u_{n}} \\
O_{u_{0}} & \ldots & O_{u_{i}} & O_{u_{n}} \\
F_{u_{0}} & \ldots & F_{u_{i}} & F_{u_{n}} \\
V_{u_{0}} & \ldots & V_{u_{i}} & V_{u_{n}} \\
m_{u_{0}} & \ldots & m_{u_{i}} & m_{u_{n}}
\end{array}\right] \text { avec } i=(1 . . n) \\
M E_{S} & =\left[\begin{array}{llll}
P_{s_{0}} & \ldots & P_{s_{i}} & P_{s_{m}} \\
O_{s_{0}} & \ldots & O_{s_{i}} & O_{s_{m}} \\
V_{s_{0}} & \ldots & V_{s_{s_{i}}} & V_{s_{m}} \\
m_{s_{0}} & \ldots & m_{s_{i}} & m_{s_{m}}
\end{array}\right] \text { avec } i=(1 . . m)
\end{aligned}
$$




\subsection{Motor component}

This component corresponds to features dedicated to tasks and roles assignment during different interaction processes. It uses the shared data and applies them on special sources in the CVE via assistance functions. Special sources are objects that can be modified during the interaction process by different assistance functions dedicated to $3 \mathrm{D}$ interaction tasks. They act as a support tools for coordination. Assistance functions are functions that help manage $3 \mathrm{D}$ interaction.

\subsection{Special sources}

Particular sources are associated with functions that can be used by the motor component of the workflow to detect actions of the participants, or inform users about actions performed by other users in the CVE. Those special sources are used to coordinate 3D interaction tasks in order to predict user's interactions. The coordination process is based on positions and orientations of the users in the CVE. The workflow engine (motor component/space) receives information from the shared component that contains state vectors of users and sources. It acts on special sources to change the perception of users in the CVE. We defined five special sources:

- 3DIFocus: It corresponds to all other virtual objects with which the user can interact. Focus can be considered as a tool for direct intention and will enable filter sources that are not in the users' field of view;

- 3DINimbus: It represents a set of users with the intention to interact on a single source. It represents the group of users who might select the source;

- 3DIAura: It represents a 3D area that surrounds a virtual object and allows single or multiuser selection. The selection is possible only if the avatars of users are in the aura of the source. This aura determines users who potentially want to select the source;

- 3DIAssistant: This particular source enables a user to be assisted on specific actions that it performs. (e.g.: selection of a source).

\subsection{Assistance function}

Assistance functions are functions that operate on particular sources that can be used by the WF engine. Those functions operate with different 3D interaction tasks (navigation, selection and manipulation) and communication tasks. By acting on these special objects, the system is able to provide assistance to users and therefore coordinate their interactions in the CVE.

\section{COLLABORATIVE MANIPULATION}

We want to allow synchronous multiuser manipulation of objects through any single-user interaction technique. In this way, the transition from the single-user to multi-user manipulation can be a natural and intuitive way. Indeed, this avoids the learning of a new metaphor and an additional cognitive load for users. Moreover, traditionally when a user selects an object via a single-user interaction, the avatar of the user becomes the"parent" item and therefore inherits the movements of the avatar. However, in the multiuser case, this principle may not apply.

\subsection{Synchronous manipulation}

Our approach is to integrate concepts of classical mechanics to modelling multiuser manipulation via a mechanical system composed by mechanical joints. In our case, our mechanical system is composed by objects and avatars. In the real world, when two users move a board, the resulting movement dependents on simultaneous users actions (users are related to the board by joints). We can model this by introducing virtual joints between avatars and objects. In our case, joints between users and the object can be modelled by fixed or ball joint links. This ball joint has three degrees of freedom on its three rotation components. Transmissible efforts will be on translation components. To determine the object movement, we use forces that users perform on the attachment points of the object. The movement of the object is calculated from forces provided by users. In fact, by solving laws of dynamics relationship, we can determine accelerations in translation and rotation of the object. Mathematical data is provided by the state matrices (Force, Speed, and Virtual weight). However, this approach presents a problem that concerning the mobility. For example, if many users want to move the object, degrees of freedoms are removed according the number of users (links). Consequently, the object cannot move. To prevent that we have created a restriction function that will compute the mobility of the mechanism. The workflow can if necessary 
block the access of object by specific users or update the mechanical links between users and objects. To do that WF will colorized the aura of the object in grey.

\subsection{Assistance for collaborative manipulation}

During the manipulation of the object by users, the workflow system can manage assistance for users in order to facilitate the object manipulation. We have called it, the Manipulation Assistance Function (MAF). MAF (see figure 1) can be activated partially or fully. Similar work done on assistance during collaborative tasks has been done by [Ullah et al., 2009]. A partial activation means that specific "3DIAssistant" appear to display manipulation directions to the users. "Fully activated" means that a manipulation control system is used, in addition of assistants. This manipulation will prevent users for not provided actions by updating the mechanical joint between a user and the object (e.g. use of $1 \mathrm{DoF}$ joint instead of a $3 \mathrm{DoF})$. Some translation axis will be ignored and the object can be immobile. When activated, users' avatars become red (see figure 4).

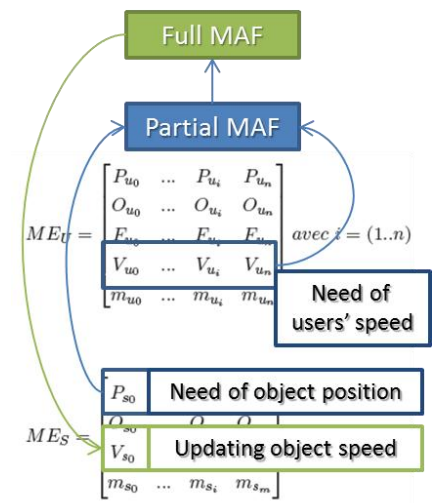

Figure 1: Use of states matrix by MAF

\section{EXPERIMENTATION}

\subsection{Overview}

This experiment is dedicated to cooperative manipulation. This multiuser experiment enables us to analyse the reaction of participants towards the use of our model and especially to study the influence of MAF on a performance of cooperative manipulation task. In this experiment, a cooperative manipulation task was carried out with a couple of users. For this purpose two Flystick devices and a simple virtual hand interaction technique were used. Figure 2 illustrates the experiment where two users manipulate a common object (a board) using two Flysticks.

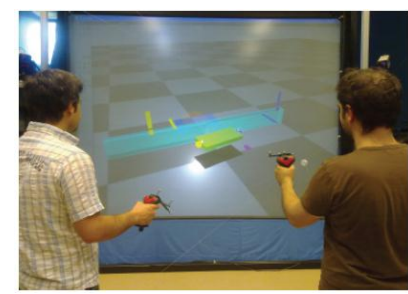

Figure 2: The VR platform where two users are performing the experiment

\section{$5.2 \quad$ Experiment specifications}

We have developed a simple collaborative virtual environment to focus on the study of the influence of the MAF on performance of a cooperative manipulation task. The aim is to compare the performances of multiuser manipulation task when the MAF is not activated (no assistance is given for coordination), partially or fully activated. Consequently, the object is already selected. In order to prepare the experiment, we have used an experiment system made by [Domingues et al., 2010]. We used a spherical joint between users and the board. The user's matrix contains their positions and orientations on the CVE, avatars weight $(1 \mathrm{~kg})$ and users' speed. Force is determined using the speed. The sources/3D objects matrix contains positions, orientations, speeds and weights of the cylinder $(0.1 \mathrm{~kg})$ and the board $(0.5 \mathrm{~kg})$.

This CVE consists of two avatars (for both users) and one board which support a free cylinder object. Users must move a board up to a drop area that will be used to validate the end of manipulation, avoiding the fall down of the cylinder that is setting above it. Two avatars (cubic shape) have the same size but with different colours. The yellow colour represents the first user, and the second user is in blue. The drop zone is in a blue colour. Access to the drop zone (target) is located behind a wall. We tested the effect of the MAF on the manipulation task performance. Three conditions were tested:

- Condition A (CA) the MAF is not activated;

- Condition B (CB) the MAF is partially activated (see figure 3);

- Condition C (CC) the MAF is fully activated (see figure 3 ). 
There were two trials under each condition and the evaluation is based on task completion time, errors and user's response collected through questionnaires. Errors are measured when the cylinder fall down. The CVE is decomposed into three sectors up to the drop zone. Sector 1 corresponds to the taking up step of the board. Sector 2 corresponds to horizontal movement of board to jump on top of the wall. Sector 3 corresponds to the go down step.

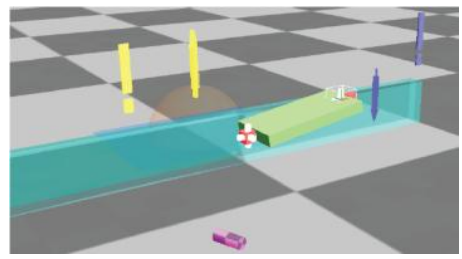

Figure 3: Full MAF: Assistants appear and manipulation control is activated. Users' avatars become red. Partial MAF: Avatars don't become red and manipulation control is not activated

\subsection{Experiment results}

This experiment (within subjects) was performed by ten couple. Participants were aged 22 to 29 years old. Each couple was given a pretrial along with a short briefing. Each couple has randomly tested all conditions two times.

\subsubsection{Errors}

The manipulation error rate corresponds to the average number of falls of cylinder of all users and for all tests. Generally, we notice that when the MAF is activated, fully or partially (CC and $\mathrm{CB}$ respectively), thus may limit the number of falls. Indeed, the visual aid is a tool for anticipating the fails; it therefore allows users to correct their manipulation strategy. Besides adding correction movements (full assistance in CC) carried out by two users allows stabilizing the board to avoid as far as possible the fall of the cylinder. Manipulation errors are more numerous on sectors 1 and 3 on CA and CB trials. Sectors 1 and 3 correspond to vertical steps and need a good coordination between users. In sector 2 , there are important manipulation errors for $\mathrm{CC}$ trials (horizontal step). We have identified two reasons which are compatible with our observations. First of all, during CA and CB trials, the cylinder often falls in sector 1 . In our protocol, it is specified that the cylinder is not repositioned on the board after the fall. He could have fallen in sector 2 . Secondly, when the board is stopped by users, the board speed is instantly passing to zero. However, the cylinder will have a low speed due to dry friction. Full MAF function does not compensate sudden board stops of the board, which means that the sector 2 is the most sensitive for this condition.

\subsubsection{Task completion time}

Trials on $\mathrm{CC}$ are faster than those of the $\mathrm{CB}$ (see figure 4). Indeed, visual assistances can certainly avoid falling objects while viewing how to correct the board movement more easily, but assistance is done at the expense of the manipulation time.

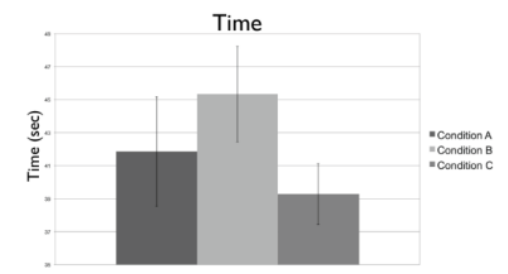

Figure 4: Influence of MAF on completion time

Indeed, we observed that in the trials under $\mathrm{CB}$ when the visual aids appears (which means a future possible fall), users take more time to talk and discuss policy to correct and avoid falling, consequently they spend more time to achieve the task. Without assistance (CA), users are not informed of a possible fall down of the cylinder and discover it only when it starts to roll on the board, which generally causes the fall of the cylinder. However, in the condition A, users do not stop working but try to avoid falling and communicate more. The full activation of the MAF provides a best time performance $(39.2 \mathrm{sec})$ comparing to $45.2 \mathrm{sec}$ and $41.4 \mathrm{sec}$ in $\mathrm{CB}$ and CA respectively. Figure 5 illustrates average time in different sectors according three conditions. CC permits to reduce total manipulation time due to the activation of full MAF. CA and CB are very similar. However, $\mathrm{CB}$ time is bigger than $\mathrm{CA}$ time on sector 2. When partial MAF is activated (CB), users will stop their actions are communicates in order to correct their moves. Consequently, CB time is superior to CA time.

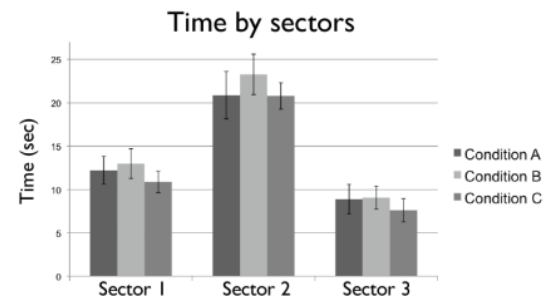

Figure 5: Average time by conditions and sectors 


\subsubsection{Users' comments and subjective analysis}

Users's comments and subjective analysis (analysis of questionnaires) revealed us the preference of users for the condition $\mathrm{C}$ in which the two kinds of assistance are available (the MAF is fully activated). This mode of operation facilitates the board movement in sectors 1 and 3 . However in sector 2, it produces cylinder falling (this requires a modification of the experiment scenario to take into account the immediate stop of the board). The use of assistant tools doesn't cause additional difficulties for users. Indeed, visual guides are comprehensible for all subjects and are found to help much during the coordination.

\subsection{Experiment conclusion}

This experiment has permitted to obtain interesting results which highlight the importance of the MAF functions (full or partial MAF). CC (full MAF) has a lower error rate and a completion time than $\mathrm{CA}$ and $\mathrm{CB}$. $\mathrm{CA}$ and $\mathrm{CB}$ have worst results on sectors 1 and 2 (vertical steps). However, our protocol is designed to allow users to continue the trial even if the cylinder falls down. We think that when the cylinder is fallen, the two users will go faster to finish the trial.

\section{CONCLUSION}

We presented a workflow-based approach to assist the coordination of 3D interactions in CVE. We highlighted the ability of the system to provide assistance to improve performances as well as in single-user interaction and in multiuser setup (in the case of more users manipulate the same object). The proposed workflow consisted of two components. The shared component is presented as the shared data space that symbolizes the behaviour of users and sources. The motor is presented as a set of assistance functions that deal with data processing from the shared space and provides tools to assist the users. It uses the shared data and applies them via assistance functions on special sources. We studied the influence of the manipulation assistance function (MAF) when two users manipulate a common object. The obtained results were encouraged because they highlight the importance of the presence of visual cues and manipulation control assistance (when MAF is fully activated). Future work will be carried out to integrate the force feedback modality and examine its effects on cooperative task. Furthermore we will evaluate and implement the system on long distance network investigate the influence of network delay on it. We are also investigating to add more data on the matrices and a new component to manage roles and permissions for users according their human machine interfaces.

\section{REFERENCES}

Aguerreche, L., Duval, T., Lecuyer, A., 2009. Short paper: 3-hand manipulation of virtual Objects. In Joint Virtual Reality Conference of EGVE - ICAT EuroVR.

Domingues C., Otmane S., Mallem M., 2010. 3DUIEF: Towards a Framework for Easy Empirica Evaluation of 3D User Interfaces and Interaction Techniques. In the International Journal of Virtual Reality. 9(1): pp. 73-80.

Duval, T., Fleury, C., 2009. An asymmetric 2d pointer/3d ray for $3 \mathrm{~d}$ interaction within collaborative virtual environments. In Proceedings of the $14^{\text {th }}$ International Conference on $3 D$ Web Technology. Web3D'09.

Duval, T., Lecuyer, A. \& Thomas, S., 2006. Skewer: a 3D interaction technique for 2-user collaborative manipulation of objects in virtual environments. In proceedings of the 3D User Interfaces. 3DUI'06.

Greenhalgh, C., Benford, S., 1999. Supporting rich and dynamic communication in large-scale collaborative virtual environments. In Presence: Teleoperators and Virtual Environments. 8(1): 1435

Noma, M., Miyasato, T., 1997. Cooperative object manipulation in virtual space using virtual physics. In Proceeding of Dynamic System and Control. ASME 61: pp. 101-106.

Otmane, S., Ouramdane-Djerah, N., Mallem, M., 2007 Towards a collaborative 3D interaction model for cooperative design in virtual environments. In computer Supported Cooperative Work in Design. IEEE CSCWD, pp. 198-203.

Pinho, M., Bowman, D., and Freitas, C. Cooperative Object Manipulation in Collaborative Virtual Environments. Journal of the Brazilian Computer Society, 14 (2), 2008, pp. 54-67.

Ullah, S., Richard, P., Otmane, S., Naud, M., Mallem, M., 2009. Human performance in cooperative virtual environments: the effect of visual aids and oral communication. In the International Journal of Virtual Reality. 8(4): pp. 79-86. 